\title{
Huge Mesenteric Lymphangioma - A Rare Cause of Acute Abdomen
}

\author{
Abstract \\ Lymphangiomas are benign congenital \\ masses which occur most commonly in \\ head and neck of children and incidence \\ of mesenteric lymphangiomas is very \\ rare. We report such a case of huge \\ mesenteric lymphangioma in a 20 year \\ old male who presented to us with acute \\ abdomen. Pre-operative diagnosis is \\ difficult due to silent clinical course and \\ lack of awareness of the clinical and \\ morphological features of this disease. \\ Keywords: Benign tomours; Congenital \\ masses; Lymphangioma. \\ Hanish Bansal \\ Raj K Jenaw \\ Rajendra Mandia \\ Department of General Surgery, S.M.S Medical College, Jaipur, Rajasthan, \\ India \\ *For correspondence: \\ Tel: $+91-9351904500$ \\ E-mail: y2khanish@rediffmail.com
}

This article is available in Embase, Index Corpenicus, Scopus, PubsHub, Chemical Abstracts, Socolar, EBSCO, African Journal Online, African Index Medicus, Open-J-Gate, Directory of Open Access Journals (DOAJ) databases

\section{Introduction}

Lymphangiomas are benign lesions of vascular origin that show lymphatic differentiation. They occur in many anatomic locations and may have a pediatric or adult clinical presentation. Most (95\%) occur in the head and neck and axillary regions. Traditionally, lymphangiomas are classified as simple, cavernous, or cystic. The simple type is usually situated superficially in the skin and composed of small thin-walled lymphatic vessels. Cavernous type is composed of dilated lymphatic vessels and lymphoid stroma, and has a connection with various normal adjacent lymphatics while the cystic type consists of lymphatic spaces of various sizes that contain fascicles of smooth muscle and collagen bundles, but has no connection with adjacent normal lymphatics [1].

Although benign in nature, mesenteric lymphangiomas may cause significant morbidity or mortality due to their large size and critical location, when they compress the adjacent structures. The most common mode of acute presentation in children is a small bowel obstruction, sometimes associated with volvulus and intestinal infarction [2]. Intestinal obstruction related to the mesenteric cysts can be induced by compression or traction by the mass [3].

Abdominal radiograph shows a gasless, homogeneous, water-dense mass that displaces bowel loops around it [4]. Ultrasonography often

Int J Health Res, December 2010; 3(4): 195 


\section{Hansal et al}

reveals a sharply-defined cystic or multicystic mass with internal septations [5]. The typical CT scan feature of mesenteric lymphangioma is a multiloculated fluid-filled mass. Characteristic thin walls and septa also can be seen on a CT scan. MDCT with thin slice multiplanar reformation is helpful to not only diagnosis the lesion, but also to assess the complications such as volvulus. CT may add important preoperative information regarding size, anatomical location, adjacent organ involvement, and complications. One report suggested that fine needle aspiration of a milky fluid containing lymphoid cells could confirm a preoperative diagnosis of lymphangioma [6].

The differential diagnosis of a cystic lesion in the pelvic cavity includes reactive ascites, duplication cysts arising from the bowel or bladder, and cystic lesions of the seminal vesicle.

The optimal treatment is radical excision, even when asymptomatic [7]. It may cause infiltration of the intestine, or involvement of the main branch of mesenteric arteries or adjacent organs that necessitate segmental resection of the intestine as was done in our case [8]. Sometimes, radical resection might be technically impossible. The prognosis is excellent if resection is complete. USG is the modality of choice for follow-up, especially in children.

\section{Case Report}

A 20 year male presented with central abdominal dull aching pain for the last 15 days, abdominal distension and constipation for 5 days and two episodes of bilious vomiting and low grade fever one day prior to visit to the hospital and was admitted into the ward.

General physical examination and vital signs were normal. On examination, abdomen was distended predominantly in epigastric and left hypochondrium and no lump was palpable. There was a dull note on percussion all over the abdomen except in left hypochondrium and epigastrium and bowel sounds were also appreciable only in above described region. Per rectal and routine blood investigations were normal. X-ray Flat plate abdomen revealed multiple air fluid levels suggestive of small intestinal obstruction. Ultrasonography was suggestive of septate ascites. CT scan (Figure 1) revealed a large lobulated mass in right subhepatic space, involving mesentery, extending up to pelvic cavity and right paracolic gutter and adjacent to ascending colon and caecum, encasing superior mesenteric vessels. Provisional diagnosis of lymphangioma was made and exploratory laprotomy was planned.

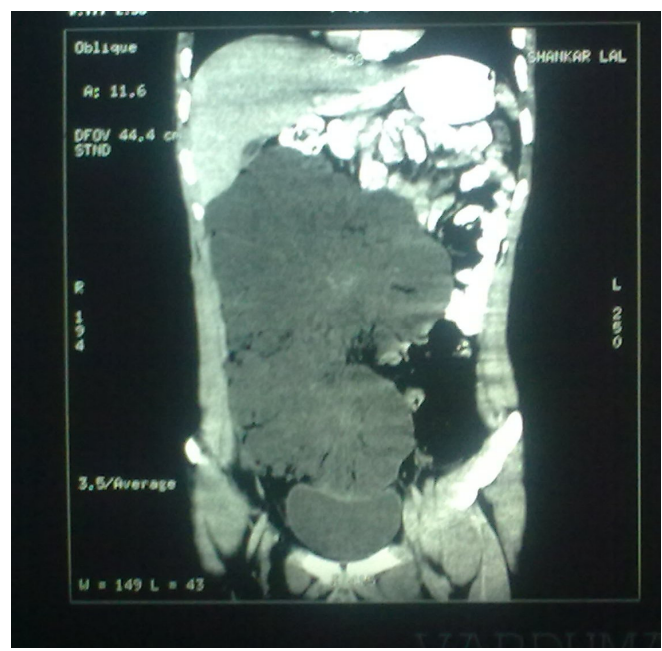

Figure 1: CT scan showing huge mass displacing small bowel.

On opening abdomen a huge grey-brown multicystic mass in mesentery was discovered (Figure 2). This was surrounding terminal ileum, ascending and proximal transverse colon and encasing superior mesenteric vessels.

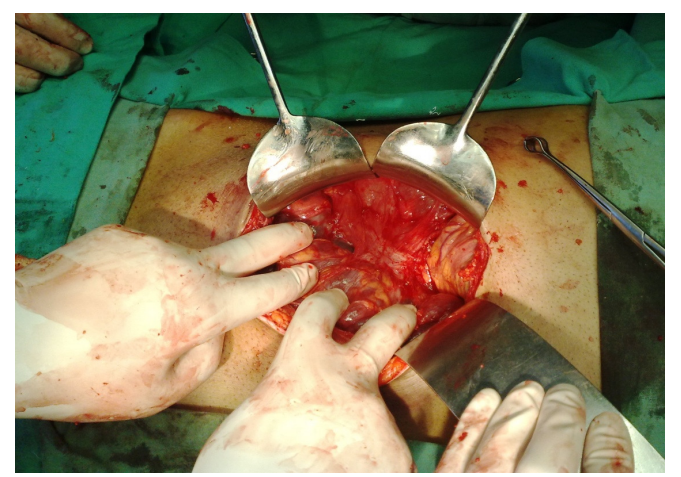

Figure 2: Per operative picture showing mesenteric lymphangioma

Int J Health Res, December 2010; 3(4): 196 


\section{Hansal et al}

Right hemicolectomy with excision of lymphangioma was done and the part adherent to superior mesenteric vessels was left unresected. Histopathology confirmed the mass as a lymphangioma measuring $20 \times 8 \times 7 \mathrm{~cm}$. The mass was multiloculated and the cut surface was spongy (Figure 3).

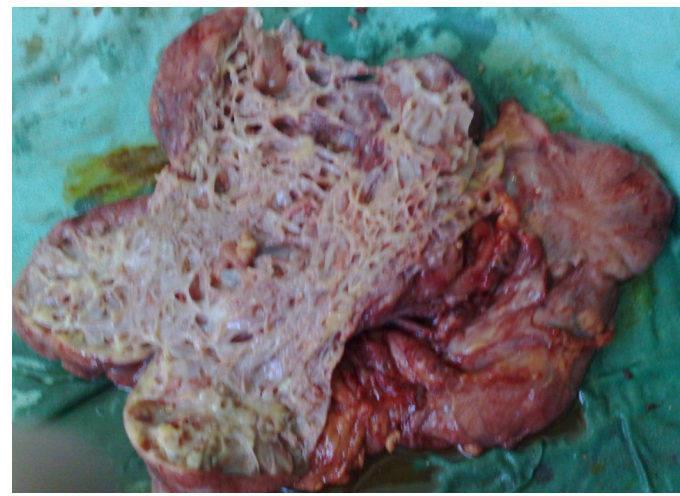

Figure 3: Cut surface of the excised mass

\section{Discussion}

This report is a case of a huge mesenteric lymphangioma which is a very rare entity. Lymphangiomas are benign lesions of vascular origin that show lymphatic differentiation. About $95 \%$ of cases occur in the head, neck and axillary regions; the remaining $5 \%$ are located in the mesentery, retroperitoneum, abdominal viscera, lung, and mediastinum [9]. The "classical presentation" is that of a low grade, partialintestinal obstruction combined with a palpable, freely-mobile abdominal mass [10]. A definite mass may be difficult to palpate because of its large size, fluid consistency, and great mobility. This case presented to us with acute intestinal obstruction without palpable lump.

The gold standard for the diagnosis of the abdominal lymphangioma in an adult is CT scan which was performed in our case. Otimal treatment is radical excision. We performed right hemicolectomy with excision of lymphangioma and a part adherent to superior mesenteric vessels was left unresected. The tumor was infiltrating into main trunk of superior mesenteric artery which is a rare entity. We could find very little literature about ways to deal with such a situation which motivated us to report this case so as to throw more light to such a scenario. Postoperative course in our case was un-eventful. In our procedure, Ryle's tube was removed on day 4 and patient was allowed orally. The patient recovery was remarkable and was discharged on 9th post operative day. A follow up CT scan after 1 year was normal without any recurrence.

\section{Conclusion}

A rare case of huge mesenteric lymphangioma has been reported in a 20 year old male. This case was successfully managed surgically with the patient making remarkable recovery..

\section{Acknowledgement}

We thank the staff of department of general surgery for their help in investigative procedure.

\section{Conflict of Interest}

No conflict of interest associated with this work.

\section{Contribution of Authors}

We declare that this work was done by the authors named in this article and all liabilities pertaining to claims relating to the content of this article will be borne by the authors.

\section{References}

1. Rieker RJ, Quentmeier A, Weiss C, Kretzschmar U, Amann K, Mechtersheimer G, et al. Cystic lymphangioma of the small-bowel mesentery. Pathol Oncol Res 2000; 6: 146-148.

2. Kosir MA, Sonnino RE, Gauderer MWL. Pediatric abdominal lymphangiomas: a plea for early recognition. J Pediatr Surg 1991; 26:1309-13.

3. Kovalivker M, Mitovic A. Obstruction and gangrene of bowel with perforation due to a mesenteric cyst in a newborn. J Pediatr Surg 1987;22:377-378.

4. Mollitt DL, Ballantine TVN, Grosfeld JL. Mesenteric cysts in infancy and childhood. Surg Gynecol Obstet 1978; 147:182-4

5. Jain S, Upreti L, Bhargava SK Gupta R, Gupta PK. Mesenteric lymphangioma: Diagnosis by multislice spiral CT. Indian J Radiol Imaging 2002.12(4):580-582.

6. Sadola E. Cystic lymphangioma of the jejunal mesentery in an adult. J Clin Ultrasound 1987; 15: 542-543

Int J Health Res, December 2010; 3(4): 197 
Hansal et al

7. Ammar SA. Successful excision of an enormous mesenteric lymphangioma in an adult. Int J Surg 2008; 17 (1): Available from http://www.ispub. com/.../successful_excision_of_an_enormous_mes enteric_lymphangioma_in_an_adult_report_of_a_c ase_1.html

8. Underhill B.M.L. Acute intestinal obstruction due to mesentric lymphangioma. Arch Dis Child 1959;34(177): 442-443.
Mesenteric Lymphangioma

9. Levy AD, Cantisani V, Miettinen M. Abdominal Lymphangiomas: Imaging features with Pathologic correlation. Am J Roentgenol 2004;182:14851491.

10. Jang JH, Lee SL, Young MK, Chang HA, Chang ED. Small bowel volvulus induced by mesenteric lymphangioma in an adult. Korean J Radiol 200910(3): 319-322.

Int J Health Res, December 2010; 3(4): 198 